\title{
OPEN Revealing the polar nature of a ferroelectric nematic by means of circular alignment
}

\author{
Per Rudquist \\ The recent discovery of spontaneously polar nematic liquid crystals-so-called ferroelectric nematics- \\ more than a century after the first discussions about their possible existence-has attracted \\ large interest, both from fundamental scientific and applicational points of view. However, the \\ experimental demonstration of such a phase has, so-far, been non-trivial. Here I present a direct \\ method for the experimental verification of a ferroelectric nematic liquid crystal phase. The method \\ utilizes a single sample cell where the two substrates are linearly and circularly rubbed, respectively, \\ and the ferroelectric nematic phase $\left(\mathrm{N}_{\mathrm{F}}\right)$ is revealed by the orientation of the resulting disclination \\ lines in the cell.
}

The simplest nematic liquid crystal phase (N, Fig. 1a) is a three-dimensional orientationally ordered liquid. The local average direction of the molecules is denoted by the director $\mathbf{n}$, and characteristic for the $\mathrm{N}$ phase is that even if the constituent (usually rodlike) molecules may be polar themselves, $\mathrm{n}$ is invariant under sign reversal, i.e., $\mathbf{n}=-\mathbf{n}$. The structure and non-polar symmetry of the $\mathrm{N}$ phase is well-known since more than a century and the electrooptic effects of nematic liquid crystals are the basis for the multi-billion dollar liquid crystal display technology.

In 2017 Nishikawa et al. ${ }^{1}$ and Mandle et al. ${ }^{2,3}$, reported materials which apparently exhibited nematic phases where locally the sign-invariance of $\mathbf{n}$ was not valid. Nishikawa et al., reported a phase transition between two fluid nematic phases in the highly polar compound DIO. In the lower temperature phase, they observed macroscopic polar ordering, probed by means of SHG measurements, in response to an applied electric field. They suggested the dipolar molecular associations to be "ferroelectric-like". Mandle et al. proposed that the local polar order in the material RM734 was driven by spontaneous splay, (splay nematic phase) which resulted a periodic structure, with alternating direction of splay and polarization ${ }^{2-4}$. Later, Sebastián et al., by means of second harmonic generation imaging, could draw the conclusion that the local order in the "splay nematic phase" of RM734 is polar on a macroscopic scale and that the splay and polarization modulation period is $5-10 \mu \mathrm{m}$. They also raised the important question whether a homogeneous uniaxial ferroelectric nematic phase can be realized, or if ferroelectric ordering in a nematic phase must be accompanied by orientational deformation ${ }^{5}$.

In 2020, Clark and co-workers reported experimental evidence that the new liquid crystal phase in RM734, first synthesized in 2017 by Mandle, Cowling and Goodby ${ }^{2-4}$, is the ferroelectric nematic $\left(\mathrm{N}_{\mathrm{F}}\right)$ phase $^{6}$, cf. Figure $1 \mathrm{~b}$. The possible existence of such a spontaneously polar nematic phase, driven by dipolar ordering of molecular dipoles in highly polar materials, was proposed already by Born in $1916^{7}$ but evidence for its existence has not been presented before. The discovery of the $\mathrm{N}_{\mathrm{F}}$ phase "opens another chapter in condensed matter science and technology" (Lavrentovich ${ }^{8}$ ) and has sparked an enormous interest, both from fundamental physics and from applicational points of view.

To understand the mechanisms behind the formation of the new $\mathrm{N}_{\mathrm{F}}$ phase, and to explore its future application potential, there is an immediate need to design, synthesize and investigate a vast number of similar materials, and rapidly scan these for the $\mathrm{N}_{\mathrm{F}}$ phase. In this letter I present a surprisingly simple and fast method for direct experimental identification of the $\mathrm{N}_{\mathrm{F}}$ phase, utilizing only a single sample cell and an optical microscope (or even the bare eye). No external electric or magnetic fields, no second harmonic generation experiments, nor any spectroscopic methods are needed. The study solely treats the experimental identification of the $\mathrm{N}_{\mathrm{F}}$ phase, with no ambition at this stage, to further elaborate on the mechanisms behind the formation of the $\mathrm{N}_{\mathrm{F}}$ phase.

In a nematic liquid crystal display cell, the quiescent state is governed by the boundary conditions at the two cell substrates. For homogeneous planar anchoring of $\mathbf{n}$ mechanically rubbed polyimide layers can be used, forcing $\mathbf{n}$ to align with the rubbing direction at the surfaces. In the conventional $\mathrm{N}$ phase, parallel, as well as 


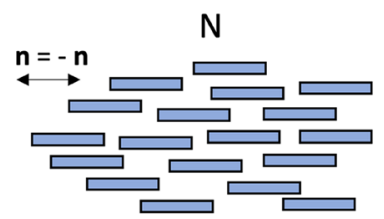

a

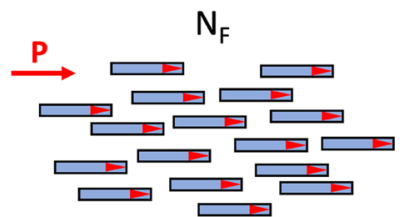

b

Figure 1. Schematic representations of $(\mathbf{a})$ the nematic $(\mathrm{N})$ and $(\mathbf{b})$ the ferroelectric nematic $\left(\mathrm{N}_{\mathrm{F}}\right)$ phases.

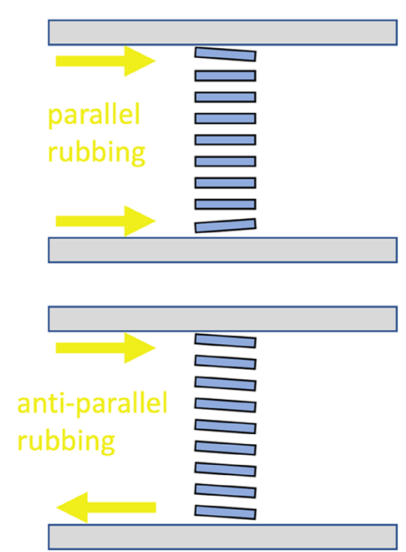

a

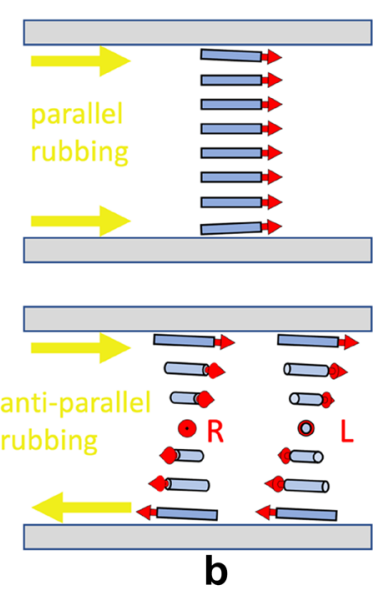

b

Figure 2. In the $\mathrm{N}$-phase (a), parallel and anti-parallel rubbing gives a non-twisted structure. In the $\mathrm{N}_{\mathrm{F}}$-phase (b) the polarization (red) aligns with the rubbing direction. In the case of anti-parallel rubbing the structure has to make a right-handed (R) or left-handed (L) twist of $180^{\circ}$ to match the in-plane polar boundary conditions $s^{9,10}$.

antiparallel, rubbing at the two surfaces produce a non-twisted director field, Fig. 2a, and if the rubbing directions are not colinear we get a director twist between the plates.

For the polar $\mathrm{N}_{\mathrm{F}}$ phase the situation is fundamentally different: on a rubbed surface the $\mathrm{N}_{\mathrm{F}}$ phase aligns with the spontaneous polarization parallel to the rubbing direction ${ }^{9}$. The striking consequence is that when the rubbing directions are anti-parallel, we get $\mathrm{a} \pm 180^{\circ}$ director twist between the surfaces, cf. Figure $2 \mathrm{~b}$. When the angle between the two rubbing directions is neither 0 nor $180^{\circ}$, the $\mathrm{N}_{\mathrm{F}}$ phase should adopt the state with the smallest twist matching the rubbing directions. When the two surfaces are rubbed in parallel we get a non-twisted state (just as in the $\mathrm{N}$ phase). The possible twisted states and electrooptic effects in the polar nematic phase are further discussed by Caimi et al. ${ }^{10}$ and Sebastián et al. ${ }^{11}$.

Let us now make a cell where the bottom surface is linearly rubbed, and the top surface is circularly rubbed. Such circularly rubbed cells (CRCs) have been used by Suh et al. to measure the pitch in long pitch cholesteric materials ${ }^{12}$. Linear alignment is obtained by gently rubbing the polyimide coated surface with a velvet cloth in a single direction. Circular alignment can be realized by putting the polyimide-coated substrate on a spinner chuck and gently press the rubbing cloth towards the spinning substrate for a few seconds. Alternatively, as in this study, a small piece of rubbing cloth can be attached to a flat rotating chuck, that is gently pressed towards to a then fixed substrate. As an alternative to mechanical rubbing, photobuffing ${ }^{13}$ could likely be used provided that the pretilt, necessary for the effective in-plane polar surface anchoring, can be secured also in circular alignment.

In a CRC the $\mathrm{N}$ phase adopts the configuration illustrated in Fig. $3 \mathrm{a}^{12}$. If we use a clockface notation and the bottom substrate is rubbed along the 3-9 direction, we get a non-twisted director field at " 12 " and " 6 ". Away from the " 12 " and " 6 " positions we get an increasing twist and at 3 and at 9 the twist changes sign, to minimize the twist elastic energy. The discontinuous change from $+90^{\circ}$ to $-90^{\circ}$ twist occurs across two sharp disclination lines running horizontally, i.e. in the " 9 "- “ 3 " orientation through the center of circular rubbing.

Let us now consider the case of the $\mathrm{N}_{\mathrm{F}}$ phase in the same cell, cf. Fig. $3 \mathrm{~b}$. The circular rubbing is made counterclockwise, and the rubbing directions are therefore parallel at " 12 " and antiparallel at " 6 ". The polar $\mathrm{N}_{\mathrm{F}}$ phase orients with the polarization along the rubbing directions and to match the boundary conditions, we expect a non-twisted director at " 12 " where the rubbing directions are in parallel. When going away from " 12 " towards " 6 ", clockwise or anticlockwise, there is a twist that increases continuously all the way to the " 6 " position, where the rubbing directions become perfectly antiparallel. To minimize the elastic energy, the twist discontinuously changes from $180^{\circ}$ right-handed to $180^{\circ}$ left-handed at " 6 " across a disclination line, while the structure is continuous at all other positions in the cell. To summarize: in the CRC cell the $\mathrm{N}$ phase should make two disclination lines along the linear rubbing direction, while the $\mathrm{N}_{\mathrm{F}}$ should make one disclination line normal to the linear rubbing direction, starting from the center of rubbing. 

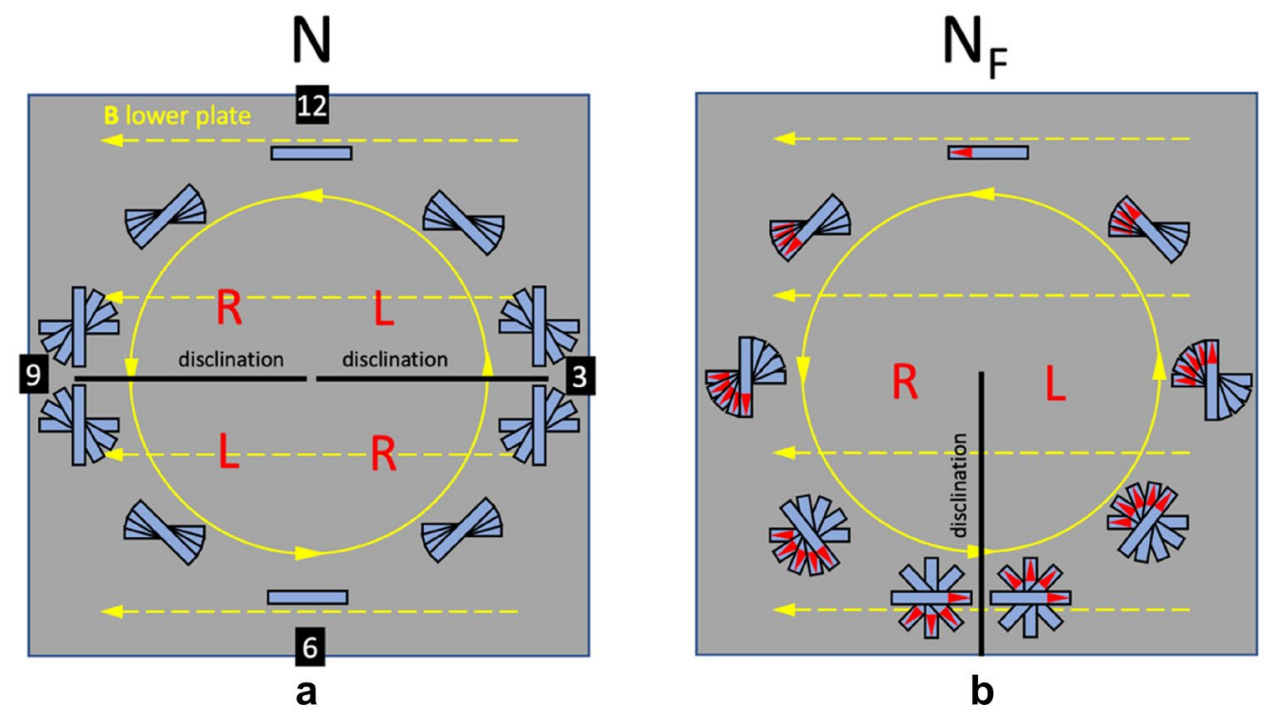

Figure 3. Schematics of circularly rubbed cells filled with (a) non polar $\mathrm{N}$, and (b) polar $\mathrm{N}_{\mathrm{F}}$ nematic liquid crystal. The $\mathrm{N}$ phase gives a disclination line parallel to the linear rubbing direction $\mathrm{B}$, while the $\mathrm{N}_{\mathrm{F}}$ phase gives a disclination line normal to $\mathbf{B}$, starting at the center of circular rubbing. $\mathrm{R}$ and $\mathrm{L}$ denote right-handed and lefthanded twisted regions, respectively.

Figure 4 shows polarized optical microscope photographs of a $4 \mu \mathrm{m}$ thick CRC filled with the archetype $\mathrm{N}_{\mathrm{F}}$ material RM734 having a phase transition between the ferroelectric $\mathrm{N}_{\mathrm{F}}$ and the conventional $\mathrm{N}$ phase at about $133^{\circ} \mathrm{C}$, and between $\mathrm{N}$ and isotropic at $188^{\circ} \mathrm{C}$, respectively. The polyimide aligning layer on both surfaces is PI2610 from DuPont, and the bottom linearly rubbed substrate is the warmer substrate (in direct contact with the hot- plate). The cell was capillary filled with RM734 in the isotropic phase at $190^{\circ} \mathrm{C}$. At $140{ }^{\circ} \mathrm{C}(\mathbf{a}$ and $\mathbf{l})$ ) we have two horizontal disclination lines emanating from the center of circular rubbing, as expected from the nonpolar N phase. At " 12 " and " 6 " on the clockface we get extinction of light as the director here is parallel to one of the crossed polarizers. At $130^{\circ} \mathrm{C}(\mathbf{f})$ the two horizontal disclination lines have been replaced by one disclination line at " 6 " which proves that the sample is now in the $\mathrm{N}_{\mathrm{F}}$ phase, cf. Fig. 3 . Figure $4 \mathrm{a}-\mathrm{f}$ shows the evolution of the cell on cooling from $140^{\circ}$ to $130^{\circ} \mathrm{C}$ at a rate of $3{ }^{\circ} \mathrm{C} / \mathrm{min}$. Soon after the phase transition (b) the two disclination lines at " 9 " and at " 3 " start to move downwards and become one disclination at " 6 ". On heating from 130 to $140{ }^{\circ} \mathrm{C}$ (Fig. 4g-l) the single disclination line at " 6 ", soon after the transition to the N phase (h), splits close to the center of circular rubbing and branches symmetrically into the two horizontal disclinations characteristic of the $\mathrm{N}$ phase at the " 9 " and " 3 " positions (1). The course of events shown in Fig. 4 is perfectly reversible.

The observed defect behavior at the $\mathrm{N}-\mathrm{N}_{\mathrm{F}}$ transition in CRC cells described here can be compared to the behavior of chiral $\mathrm{N}_{\mathrm{F}}$ materials in so-called Cano-wedge cells ${ }^{14,15}$. In chiral nematics $\left(\mathrm{N}^{*}\right)$ the director forms a spontaneous twist with a helix axis normal to the director. In a wedge cell with homogenous planar anchoring, a periodic set of disclination lines appears, normal to the opening direction of the wedge. At each disclination the number of half-pitches increases in steps of one to minimize the elastic energy. In a polar and chiral $\mathrm{N}_{\mathrm{F}}{ }^{*}$ phase where $\mathbf{n} \neq-\mathbf{n}$, the number of half-pitches instead increases by two in each disclination in such wedge cells ${ }^{14,15}$. Hence the number of disclination lines are then reduced by a factor of 2 at the transition between $\mathrm{N}^{\star}$ and $\mathrm{N}_{\mathrm{F}}^{*}$. However, in the Cano-wedge case the material must be chiral to show this defect behavior. Furthermore, if the material does not exhibit both the $\mathrm{N}^{\star}$ phase and the $\mathrm{N}_{\mathrm{F}}^{*}$ phase in the sequence, one cannot, merely based on the number of disclination lines in the wedge, draw any conclusion about the polarity of the nematic phase under study. In contrast, the CRC method described here works for both achiral and chiral versions of the $\mathrm{N}_{\mathrm{F}}$ phase, and importantly, regardless of whether the $\mathrm{N}$ phase is present or not in the phase sequence. Hence, in liquid crystals with a direct phase transition from isotropic to ferroelectric nematic, see for instance Manabe et al. ${ }^{16}$ and $\mathrm{Li}$ et al. ${ }^{17}$, the configuration with one disclination line should still form in CRC cells, revealing the polar order of the $\mathrm{N}_{\mathrm{F}}$ phase. It is also worth pointing out that the CRC cell method should in principle facilitate pitch measurements of the chiral $\mathrm{N}_{\mathrm{F}}{ }^{*}$ phase, where the pitch in can be calculated from the azimuthal rotation of the single disclination line away from the " 6 " position, analogous to the pitch measurements of the conventional $\mathrm{N}^{\star}$ phase in CRC described by Suh et al. ${ }^{12}$.

To conclude, circularly rubbed cells and the position of the disclination line(s) in relation to the linear rubbing direction constitute a simple and yet powerful tool to probe the polar order of nematic liquid crystals, and in particular to unambiguously distinguish the $\mathrm{N}_{\mathrm{F}}$ phase from the $\mathrm{N}$ phase.

The data that supports the findings of this study are available within the article. 

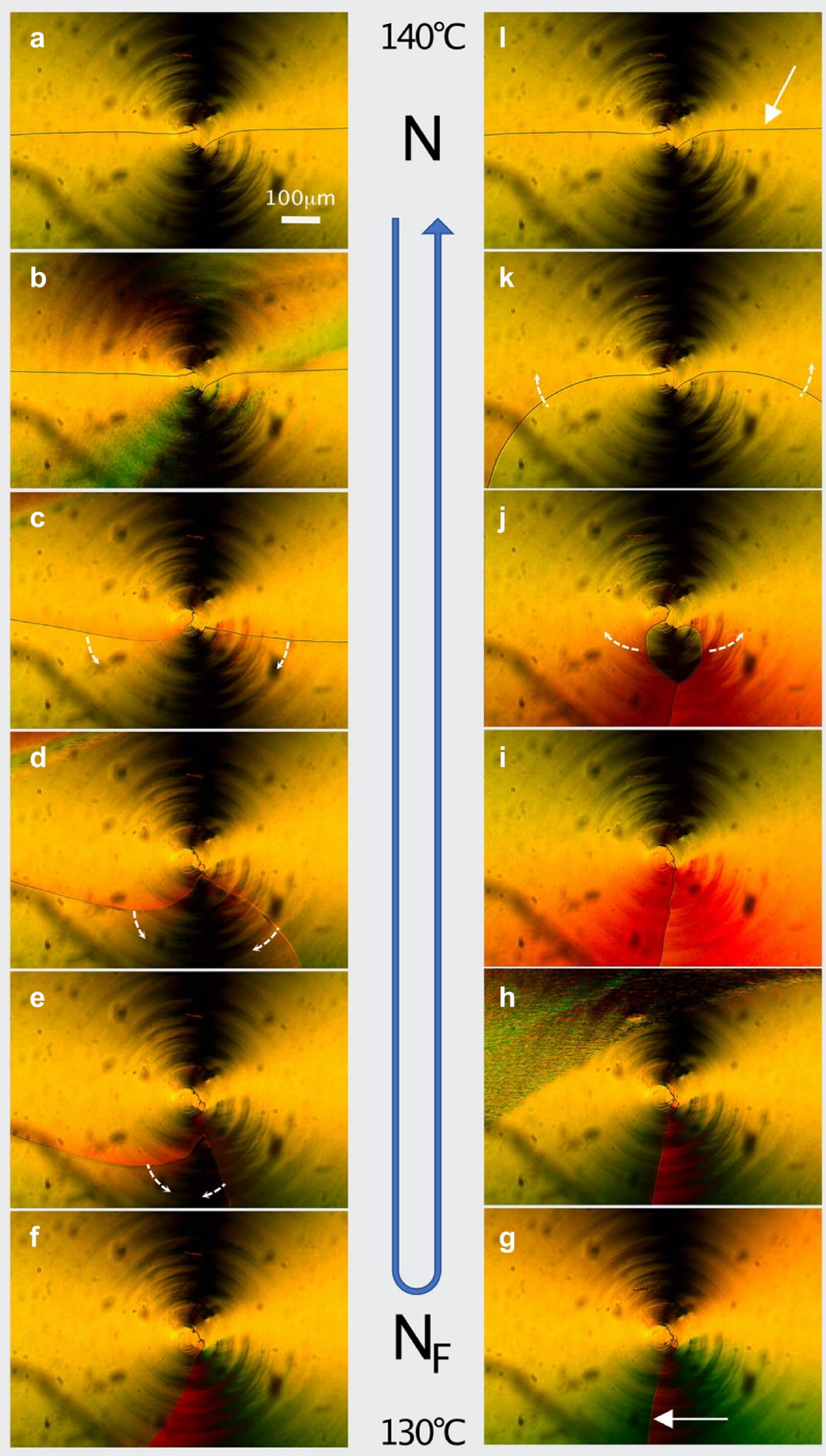

Figure 4. Evolution of the CRC structure of RM734 on cooling from $140^{\circ} \mathrm{C}$ to $130^{\circ} \mathrm{C}(\mathbf{a}-\mathbf{f})$ and on heating from $130^{\circ}$ to $140^{\circ} \mathrm{C}(\mathbf{g}-\mathbf{l})$. The disclinations and their direction of motion are marked with solid and dashed arrows, respectively. In $\mathbf{b}$ and $\mathbf{h}$, the regions with irregular texture appear during the phase transition which occurs at approximately the same temperature (at " $\left.133^{\circ} \mathrm{C}\right)$ on both cooling and heating. The faint circular stripes are due to scratches in the alignment layer, caused by mechanical unevenness during the circular rubbing process. Hence, these stripes visualize the rubbing orientation on the circularly rubbed top plate. 
Received: 18 October 2021; Accepted: 14 December 2021

Published online: 23 December 2021

\title{
References
}

1. Nishikawa, K. et al. A fluid liquid crystal material with highly polar order. Adv. Mater. 29, 1702354 (2017).

2. Mandle, R. J., Cowling, S. J. \& Goodby, J. W. Rational design of rod-like liquid crystals exhibiting two nematic phases. Chemistry 23, 14554-14562 (2017).

3. Mandle, R. J., Cowling, S. J. \& Goodby, J. W. A nematic to nematic transformation exhibited by a rod-like liquid crystal. Phys. Chem. Chem. Phys. 19, 11429-11435 (2017).

4. Mertelj, A. et al. Splay nematic phase. Phys. Rev. X 8, 041025 (2018).

5. Sebastián, N., Cmok, L., Mandle, R. J., de la Fuente, M. R., Drevenšek Olenik, I., Copic, M., \& Mertelj, A. Ferroelectric-Ferroelastic phase transition in a nematic liquid crystal. Phys. Rev. Lett. 124, 037801 (2020).

6. Chen, X. et al. First-principles experimental demonstration of ferroelectricity in a thermotropic nematic liquid crystal: polar domains and striking electro-optics. PNAS 117, 14021-14031 (2020).

7. Born, M. Über anisotrope Flüssigkeiten. Versuch einer Theorie der flüssigen Kristalle und des elektrischen Kerr-Effekts in Flüssigkeiten. Sitzungsber. Preuss. Akad Wiss. 30, 614-650 (1916)

8. Lavrentovich, O. Ferroelectric nematic liquid crystal, a century in waiting. PNAS 117(26), 14629-14631 (2020).

9. Chen, X. et al. Polar in-plane surface orientation of a ferroelectric nematic liquid crystal: Polar monodomains and twisted state electro-optics. PNAS 118(22), e2104092118 (2021).

10. Caimi, F. et al. Surface alignment of ferroelectric nematic liquid crystals. Soft Matter 17, 8130-8139 (2021).

11. Sebastián, N., Mandle, R. J., Petelin, A., Eremin, A., Mertelj, A. Electrooptics of mm-scale polar domains in the ferroelectric nematic phase Liquid Crystals 2021, AHEAD-OF-PRINT, 1-17 https://doi.org/10.1080/02678292.2021.1955417

12. Suh, S., Joseph, K., Cohen, G., Patel, J. S. \& Lee, S.-D. Precise determination of the cholesteric pitch of a chiral liquid crystal in a circularly aligned configuration. Appl. Phys. Lett. 70, 2547 (1997).

13. Yaroshchuk, X. O. \& Reznikov, Y. Photoalignment of liquid crystals: basics and current trends. J. Mater. Chem. 22, 286-300 (2012).

14. Zhao, X., Zhou, J., Li, J., Kougo, J., Wan, Z., Huang, M. \& Aya, S. Spontaneous helielectric nematic liquid crystals: Electric analog to helimagnets. PNAS 118(42), e2111101118 (2021).

15. Nishikawa, H., \& Araoka, F. A new class of chiral nematic phase with helical polar order. Adv. Mater., 33, 2101305 (2021).

16. Manabe, M. \& Bremer, M. Kraska Ferroelectric nematic phase at and below room temperature. Liq. Cryst. 48(8), 1079-1086 (2021).

17. Li, J. et al. How far can we push the rigid oligomers/polymers toward ferroelectric nematic liquid crystals. J. Am. Chem. Soc., 143(42), 17857-17861 (2021).

\section{Acknowledgements}

The $\mathrm{N}_{\mathrm{F}}$ material RM734 was kindly supplied by E. Korblova and D. M. Walba, at University of Colorado at Boulder, USA.

\section{Author contributions}

P.R. proposed the method, carried out the experiments, analysed the results, and wrote the paper.

\author{
Funding \\ Open access funding provided by Chalmers University of Technology.
}

\section{Competing interests}

The authors declare no competing interests.

\section{Additional information}

Correspondence and requests for materials should be addressed to P.R.

Reprints and permissions information is available at www.nature.com/reprints.

Publisher's note Springer Nature remains neutral with regard to jurisdictional claims in published maps and institutional affiliations.

Open Access This article is licensed under a Creative Commons Attribution 4.0 International License, which permits use, sharing, adaptation, distribution and reproduction in any medium or format, as long as you give appropriate credit to the original author(s) and the source, provide a link to the Creative Commons licence, and indicate if changes were made. The images or other third party material in this article are included in the article's Creative Commons licence, unless indicated otherwise in a credit line to the material. If material is not included in the article's Creative Commons licence and your intended use is not permitted by statutory regulation or exceeds the permitted use, you will need to obtain permission directly from the copyright holder. To view a copy of this licence, visit http://creativecommons.org/licenses/by/4.0/.

(C) The Author(s) 2021 